\title{
COMBINED FUNCTION MAGNET PROFILE DESIGN
}

\section{S.C. Snowdon}

November 1983

\section{INTRODUCTION}

Conformal mapping has been used in the past to generate magnet poleface contours. ${ }^{1}$ The present effort extends a notion considered previously in which a plane of symmetry was introduced. One further mapping permits the plane of symmetry to be transformed into a curved surface of bifurcation. A magnetostatic problem set on one side may be continued into the other side thereby providing an exact solution on both sides. An application is made for the Beijing Synchrotron ${ }^{2}$ booster magnet.

\section{COMBINED FUNCTION MAGNET}

The desired poleface contour is considered to be one of the surfaces for which the magnetostatic potential $V$ is a constant. As $V$ approaches some limit $V_{0}$ the contour is usually reduced to a sequence of straight lines in the z-plane which are then related to the real axis of the s-plane by a suitable transformation. ${ }^{3}$ For a combined function magnet the previous $z-p l a n e$ is designated as the $r$-plane and a transformation is made to the $z-p l a n e$ using $r=z+\frac{1}{2} k z^{2}+\frac{1}{6} k^{1} z^{3}$. This distorts the generator elements $\left(V=V_{0}\right)$ from linear to curved segments. The mappings are illustrated in Figures $(1-3)$.

Quantitatively one bends the real axis of the s-plane into a polygon whose interior angles are indicated in Figure 1. The result in the $r$-plane may be written as ${ }^{4}$ 


$$
r=\frac{q_{1}}{C_{1}} \int \frac{\left(1+\frac{1}{\alpha}\right)\left(1+\frac{s}{\gamma}\right)}{(1+s)^{2-\frac{\omega}{\pi}}\left(1-\frac{s}{\delta}\right)^{\frac{1}{2}}\left(s^{2}-\tau^{2}\right)^{\frac{1}{2}}} d s,
$$

where $-\alpha,-1,-\gamma,-\tau, \tau$, and $\delta$ are bend points in the s-plane. For the magnetostatic problem shown in Figure 1 where $U$ is the stream function and $V$ is the potential function, the result of transforming to the $r$-plane represents a symmetrical dipole and is shown in Figure 2.

To produce the right hand side of a combined function magnet the $r$-plane is distorted into the z-plane using

$$
r=z+\frac{1}{2} k z^{2}+\frac{1}{6} k^{\prime} z^{3}
$$

where $k$ is the field index and $k^{\prime}$ is the gradient index.

Magnetostatic considerations are readily inserted into a $w-p l a n e$ which is obtained from the $s-p l a n e$ using bends only at $\pm \tau$. Thus

$$
w=\int \frac{d s}{\left(s^{2}-\tau^{2}\right)^{\frac{1}{2}}} \text { or } \quad s=\tau \cos (i w) \text {. }
$$

From Figure 4 it may be seen that a uniform field in the w-plane yields an excitation in the s-plane in which the exciting currents are at infinity. The complex potential is

$$
W=U+i V=\frac{V_{0}}{\pi} w
$$

This representation of the complex potential will be modified later in order to locate the excitation current at a finite distance.

Starting with the w-plane and defining for convenience

$$
G(s)=\frac{(1+s)^{2-\frac{\omega}{\pi}}\left(1-\frac{s}{\delta}\right)^{\frac{1}{2}}}{\left(1+\frac{s}{\alpha}\right)\left(1+\frac{s}{\gamma}\right)}
$$


one has

$$
r=\frac{q_{1}}{C_{1}} \int \frac{d w}{G(s)}
$$

and for the magnetic field

$$
H^{*}=H_{x}-i H_{y}=i \frac{d W}{d z}=i \frac{V_{0}}{\pi} \cdot \frac{C_{1}}{g_{1}} G(s) \cdot\left[1+k z+\frac{i}{2} k^{i} z^{2}\right] .
$$

The constant $q_{1}$ as shown in Figure 2 is the imaginary part of $r$ on the surface $V=V_{1}$ associated with the curve of bifurcation $U=0$. By integrating in Eq. (6) along the line $\rho=0$ from $\phi=0$ to $\phi=\phi_{1}$ one obtains $C_{\eta}$. Thus

$$
C_{i}=\int_{0}^{\phi_{i}} \frac{d \varphi}{G(\xi)},
$$

where

$$
\xi=\tau \cos \varphi
$$

Equation (7) may be used to relate $V_{0}$ to an assigned magnetic induction $B_{0}$ at the origin $x=0, y=0$

$$
H_{y}(0,0)=-\frac{V_{0}}{\pi} \frac{C_{1}}{g_{1}} G(\tau)=-B_{0}
$$

To find the magnetic field gradient on the median plane differentiate Eq. (7) with respect to $z$, set $y=0$ and take the imaginary part. Thus

$$
\frac{H_{y}^{\prime}(x)}{H_{y}(0)}=\frac{G(\xi)}{G(\tau)}\left\{k+k^{\prime} x+\left(1+k x+\frac{1}{2} k^{\prime} x^{2}\right)^{2} \frac{C_{i}}{g_{1}} \sqrt{\xi^{2}-\tau^{2}} G(\xi) \frac{d \ln G}{d \xi}\right\}
$$

where from Eq. (6)

$$
p=\frac{q_{1}}{C_{1}} \int_{0}^{p} \frac{d p}{G(\xi)}
$$


with

$$
\xi=\tau \cosh \rho
$$

and from $\mathrm{Eq} .(2)$

$$
p=x+\frac{1}{2} k x^{2}+\frac{1}{6} k^{\prime} x^{3}
$$

The contour represented by $V=V_{1}$ or $\phi=\phi_{1}$ may be found using Eq. (6) with

$$
s=\tau \cos \left[i\left(\rho+i \phi_{1}\right)\right]
$$

Thus

$$
r=i q_{1}+\frac{q_{1}}{C_{1}} \int_{0}^{p} \frac{d p}{G(s)},
$$

where $r$ is related to $z$ by Eq. (2). If Eq. (7) is evaluated for values of $s$ in Eq. (15) the magnetic field may be found along the contour. Note that $\rho$ is a convenient variable for parametrizing all variables of interest. Any method of numerical integration of Eqs. (8), (12), and (16) wit1 yield results for the desired quantities provided that $B_{0} k k^{4} q_{1} \omega^{\omega} \phi_{1} \delta$ $\alpha \gamma$ and $\tau$ are given. However, the design problem is to find these constants in terms of physically specifiable characteristics of the magnet and its field. By adding two more unknowns, $\rho_{0}$ the value of $\rho$ at the maximum excursion of the gradient from the "ideal" gradient, and $\rho_{e}$ the value of $\rho$ at the maximum extent of the "good field" region, one may utilize information to be specified at these points. Table 1 includes a comparison of these unknowns with quantities and conditions to be specified. Note that the "ideal" field on the median plane is 


$$
H_{y}=-B_{0}\left(1+k x+\frac{1}{2} k^{\prime} x^{2}\right)
$$

and that the deviation of the actual median plane field from the "ideal" field is

$$
\frac{\Delta H_{y}}{H_{y 0}}=\left[\frac{G(\xi)}{G(\tau)}-1\right] \cdot\left(1+k x+\frac{1}{2} k^{6} x^{2}\right) .
$$

\begin{tabular}{|c|c|c|c|}
\hline Unknown & Given & Unknown & Given \\
\hline$B_{0}$ & $B_{0}$ & $\alpha$ & $\Delta H_{y}^{1} / H_{y o} \quad$ at $\rho_{0}$ \\
\hline$k$ & $k$ & $\gamma$ & $\Delta H_{v}^{\prime \prime} / H_{v 0}=0$ at $\rho_{0}$ \\
\hline$k^{\prime}$ & $k^{\prime}$ & $\tau$ & $\Delta H_{y}^{1} / H_{\text {yo }}$ at $\rho e$ \\
\hline$q_{1}$ & $\mathrm{y}_{1}$ & $\rho_{0}$ & $x \quad$ at $\rho_{e}$ \\
\hline$\omega$ & $\omega$ & $\rho_{e}$ & $\Delta H_{y}^{\prime \prime} / H_{y o}=0$ at $\rho=0$ \\
\hline$\phi$ & $\phi_{1}$ & & \\
\hline$\delta$ & $\rho_{\delta}$ & & \\
\hline
\end{tabular}

The unknowns that are directly given are $B_{0} k$ and $k^{\prime}$ which characterize the "ideal" field. The unknown $q_{1}$ may be found from the half gap $y_{1}$ using Eq. (2). Tentative choices are made for $\omega \phi_{1}$ and $\rho_{\delta}$. Generally $\omega$ must be greater than $\pi / 2$ in order to have a maximum $x$ associated with the edge of the pole. If the corresponding $y$ at this location is too large w must be increased. The value of $\phi_{1}$ must be less than $\pi$ order that the contour not 
coincide with the generator surface. For $\rho_{\delta}$ no guide is available but a number around 10 seems to correspond to reasonable yoke locations. U1timately $\phi_{1}$ and $\rho_{\delta}$ are varied to give the desired pole width and backleg location.

It is to be noted that the central gradient divided by the central field (field index) is

$$
\frac{H_{y}^{\prime}(0,0)}{H_{y}(0,0)}=k
$$

and that the gradient of the gradient of the field divided by the central field (gradient index) is

$$
\frac{H_{y}^{\prime \prime}(0,0)}{H_{y}(0,0)}=k^{\prime}+\frac{C_{j}^{2}}{g_{1}^{2}} \tau G^{2}(\tau)\left(\frac{d \ln G}{d \xi}\right)_{\xi=\tau} .
$$

Furthermore, as may be seen 1ater, Eqs. (18) and (19) are unchanged after the coils are located at finite distances. Hence it is to be noted that the calculated field index and gradient index will equal those calculated from the "ideal" field provided

$$
\left(\frac{d \ln G}{d \xi}\right)_{\xi=\tau}=\frac{2-\frac{\omega}{\pi}}{1+\tau}-\frac{\frac{1}{2}}{\delta-\tau}-\frac{1}{\alpha+\tau}-\frac{1}{\gamma+\tau}=0 .
$$

This condition is adopted as a given quantity and appears as the last entry in Table 1. If Eq. (20) is used to solve for $\gamma$ four unknowns remain: $\rho_{0}$ $\rho_{\mathrm{e}} \alpha$ and $\tau$. These may be found from the four remaining given quantities or conditions.

\section{INITIALIZATION OF PARAMETERS}

Trial values for $\rho_{0} \rho_{e}{ }^{\alpha} \tau$ are calculated from a simplified mode $1^{5}$ in which $\xi$ is considered large with respect to $x$ but smal1 with respect to 1 . 
$-7-$

For simplicity the return yoke position is removed to infinity $(\delta=\infty)$.

Expressions for the first four derivatives of the field at the origin

are identical with those of the "ideal" field if

$$
\left(\frac{d \ln G}{d \xi}\right)_{\xi=\tau}=\left(\frac{d^{2} \ln G}{d \xi^{2}}\right)_{\xi=\tau}=0 \text {. }
$$

From Eq. (20) and the assumption that $\tau$ is negligible and that $\delta=\infty$, Eq. (21) gives

$$
\frac{1}{\alpha_{0}}+\frac{1}{\gamma_{0}}=2-\frac{\omega}{\pi}, \quad \frac{1}{\alpha_{0}^{2}}+\frac{1}{\gamma_{0}^{2}}=2-\frac{\omega}{\pi} .
$$

Solving for $\alpha_{0}$ and $\gamma_{0}$ one has

$$
\frac{1}{\alpha_{0}}=\frac{1}{2}\left(2-\frac{w}{\pi}\right)\left[1-\sqrt{1-2 \frac{1-\omega / \pi}{2-\omega / \pi}}\right]
$$

and

$$
\frac{1}{\gamma_{0}}=\frac{1}{2}\left(2-\frac{\omega}{\pi}\right)\left[1+\sqrt{1-2 \frac{1-\omega / \pi}{2-\omega / \pi}}\right]
$$

$(24)$

For the excess over the "ideal" field Eq. (11) gives approximately

$$
\frac{\Delta H_{y}^{\prime}}{H_{y=}}=\frac{C_{1}}{g_{1}^{\prime}} \xi\left(\frac{2-\omega / \pi}{1+\xi}-\frac{1}{\alpha+\xi}-\frac{1}{\gamma+\xi}\right) \equiv \frac{\Delta H^{\prime}}{H} .
$$

For convenience let

$$
\xi=\eta \epsilon \quad \frac{1}{\alpha}=\frac{1}{\alpha_{0}}-\epsilon \quad \frac{1}{\gamma}=\frac{1}{\gamma_{0}}+\epsilon .
$$

Then

$$
\frac{\Delta H^{\prime}}{H}=\frac{C_{1}}{q_{i}^{\prime}} E^{3} \eta\left[-2\left(\frac{1}{\alpha_{0}}-\frac{1}{\gamma_{0}}\right) \eta+\left(2-\frac{\omega}{\pi}-\frac{1}{\alpha_{0}^{3}}-\frac{1}{\gamma_{0}^{3}}\right) \eta^{2}\right]
$$


At $\eta=\eta$

$$
\begin{aligned}
& \left(\frac{\Delta H^{\prime}}{H}\right)_{0}=\frac{C_{1}}{q_{1}^{\prime}} \epsilon^{3} \eta_{0}^{2}\left[-2\left(\frac{1}{\alpha_{0}}-\frac{1}{\gamma_{0}}\right)+\left(2-\frac{\omega}{\pi}-\frac{1}{\alpha_{0}^{3}}-\frac{1}{\gamma_{0}^{3}}\right) \eta_{0}\right], \\
& {\left[\frac{\partial\left(\frac{\Delta H^{\prime}}{H}\right)}{\partial \eta}\right]_{0}=\frac{C_{1}}{q_{1}} \epsilon^{3} \eta_{0}\left[-4\left(\frac{1}{\alpha_{0}}-\frac{1}{\gamma_{0}}\right)+3\left(2-\frac{\omega}{\pi}-\frac{1}{\alpha_{0}^{3}}-\frac{1}{\gamma_{0}^{3}}\right) \eta_{0}\right]=0,}
\end{aligned}
$$

and at $n=n_{e}$

$$
\left(\frac{\Delta H^{6}}{H}\right)_{e}=\frac{C_{1}}{g_{i}} \epsilon^{3} \eta_{e}^{2}\left[-2\left(\frac{1}{\alpha_{0}}-\frac{1}{\gamma_{0}}\right)+\left(2-\frac{\omega}{\pi}-\frac{1}{\alpha_{0}^{3}}-\frac{1}{\gamma_{0}^{3}}\right) \eta_{e}\right] .
$$

since $G(\xi) \cong 1$, Eq. (8) gives

$$
C_{1} \cong \phi_{1} \text {. }
$$

From Eq. (29)

$$
\eta_{0}=\frac{4}{3} \cdot \frac{\frac{1}{\alpha_{0}}-\frac{1}{\gamma_{0}}}{2-\frac{\omega}{\pi}-\frac{1}{\alpha_{0}^{3}}-\frac{1}{\gamma_{0}^{3}}}=\frac{8 \pi}{3 \omega} \cdot \frac{\sqrt{1-2 \frac{1-\omega / \pi}{2-\omega / \pi}}}{1-\omega / \pi} .
$$

Equation (28) may be solved for $\varepsilon$ and Eq. (30) then yields $n_{e}$ by solving a cubic. Equation (26) gives $\xi_{0}$ and $\xi_{e}$. However Eq. (12) by noting that $G(\xi) \cong 1$ gives in particular

$$
p_{e}=\frac{C_{1}}{g_{1}} p_{e} \cong \frac{p_{1}}{y_{1}} x_{e} \text {. }
$$

This together with $\xi_{\mathrm{e}}$ may be used in Eq. (13) to give an estimate of $\tau$. 
With this value of $\tau \mathrm{Eq}$. (13) may again be used to obtain $p_{0}$

$$
q_{0} \cong \ln \left(\frac{2 \xi_{0}}{\tau}\right) \text {. }
$$

Finally using $\varepsilon$ found from Eq. (28), Eq. (26) yields $\alpha$ and $\gamma$. In this manner sufficiently accurate trial values for $\rho_{0} \rho_{e} a$ and $\tau$ may be found. ITERATIVE PROCEDURE

If $F\left(\begin{array}{lll}\rho & \alpha & \tau\end{array}\right)$ designates the gradient in Eq. (11) and $p\left(\begin{array}{lll}\rho & \alpha & \tau\end{array}\right)$ is given by Eq. (12) then

$$
\begin{gathered}
F_{0}=F_{000}+\left(\frac{\partial F}{\partial \rho}\right)_{000} \Delta \rho_{0}+\left(\frac{\partial F}{\partial \alpha}\right)_{000} \Delta \alpha+\left(\frac{\partial F}{\partial \tau}\right)_{000} \Delta \tau \\
\left(\frac{\partial F}{\partial \rho}\right)_{0}=\left(\frac{\partial F}{\partial \rho}\right)_{000}+\left(\frac{\partial^{2} F}{\partial \rho^{2}}\right)_{000} \Delta \rho_{0}+\left(\frac{\partial^{2} F}{\partial \rho \partial \alpha}\right)_{000} \Delta \alpha+\left(\frac{\partial^{2} F}{\partial \rho \partial \tau}\right)_{000} \Delta \tau \\
F_{e}=F_{e 00}+\left(\frac{\partial F}{\partial \rho}\right)_{e 00} \Delta \rho_{e}+\left(\frac{\partial F}{\partial \alpha}\right)_{e 00} \Delta \alpha+\left(\frac{\partial F}{\partial \tau}\right)_{e 00} \Delta \tau \\
p_{e}=p_{e 00}+\left(\frac{\partial p}{\partial \rho}\right)_{e 00} \Delta \rho_{e}+\left(\frac{\partial p}{\partial \alpha}\right)_{e 00} \Delta \alpha+\left(\frac{\partial p}{\partial \tau}\right)_{e 00} \Delta \tau,
\end{gathered}
$$

where the values of $\rho_{0} \rho_{e}$ a $\tau$ on the right hand side have been assigned trial values. The quantities on the left hand side are specified by the designer. Generally speaking $F_{0}$ is the maximum allowed positive excursion from the "ideal" gradient and $F_{e}$ is the maximum allowed negative excursion from the "ideal" gradient. By definition $\frac{\partial F}{\partial \rho}$ is found using the "ideal" gradient at $\rho_{0}$. For example, a zero gradient magnet will have $\frac{\partial F}{\partial \rho}=0$. The value of $p$ is chosen via Eq. (14) from a value of $x$ equal to the "good field width" for the right or left hand side of the magnet which ever is under consideration. Numerical differentiation is employed 
to obtain all coefficients. Matrix inversion then gives the increments $\Delta \rho_{0} \Delta \rho_{\mathrm{e}} \Delta \alpha \Delta \tau$ which are used to obtain improved trial values. The process is repeated until a suitable convergence criterion is met.

Having found the unknowns for an assumed $\phi_{1}$ and $\rho_{\delta}$ the process is repeated for three additional cases in which $\phi_{1}$ is incremented by a small amount and $\rho_{\delta}$ incremented by a small amount. These four runs are used together with an assumed bilinear variation of $x_{\max }$ on the contour and $x_{1 e g}$ at the yoke with respect to $\phi_{1}$ and $\rho_{\delta}$ to yield new trial values for $\phi_{1}$ and $\rho_{\delta}$ that will provide given polewidths and yoke positions. This operation is repeated until the changes in $x_{\max }$ and $x_{1 \text { eg }}$ are acceptably sma 11.

After the search mode has been executed and suitable constants found the contour mode is activated by using the parameters found to calculate $z$ on the contour as a function of $p$ from Eqs. (16) and (2). On this contour the magnetic field from Eq. (7) and the flux from Eq. (4) are also found. On the median plane Eq. (7) gives the field and Eq. (11) gives the gradient each as a function of $\rho$ which in turn is converted to $x$ using Eqs. (12) and (14).

COMBINED FUNCTION MAGNET WITH COIL

In order to insert a current filament at a finite distance in the $w-p l a n e$ of Figure 4 it is convenient first to transform to the $\lambda$-plane using

$$
w=\frac{\phi_{1}}{\pi} \ln \lambda \quad \text { or } \quad \lambda=e^{\frac{\pi}{\phi_{1}}}
$$

Figure 5 illustrates the current filament at $\lambda_{0}$ and its images necessary to preserve the previous constant potential surfaces. The multivalued potential $\mathrm{V}$ is represented in the $\lambda$-plane as arising from double layers or cuts along the arcs shown. The complex potential for the current filament at $\lambda_{0}$ and its images is 
$-11-$

$$
W=\frac{V_{1}}{2 \pi} \ln \left[\frac{\left(\lambda_{0} \lambda-1\right)\left(\lambda_{0}^{*} \lambda-1\right)}{\left(\lambda-\lambda_{0}\right)\left(\lambda-\lambda_{0}^{*}\right)}\right]
$$

If $\lambda_{0} \rightarrow \infty$ Eq. (39) is seen to approach Eq. (4) since from Figure 4 the fraction $v_{1} / V_{0}$ is equal to $\phi_{7} / \pi$.

For a uniform current density block Eq. (39) may be generalized to

$$
W=2 J_{0} \iint \ln \left[\frac{\left(\lambda_{0} \lambda-1\right)\left(\lambda_{0}^{*} \lambda-1\right)}{\left(\lambda-\lambda_{0}\right)\left(\lambda-\lambda_{0}^{*}\right)}\right] d x_{0} d y_{0}
$$

at least when $\lambda$ refers to a location outside of the current block which is the present range of interest. The current density is

$$
J_{0}=\frac{V_{1}}{4 \pi A}
$$

where $A$ is the cross sectional area of the block.

Beth $^{6}$ has shown that if $h(z)$ is analytic

$$
\iint h(z) d x d y=\frac{1}{2 \dot{2}} \oint h(z) z^{*} d z,
$$

from which it also follows that

$$
\iint h\left(z^{*}\right) d x d y=-\frac{1}{2 \dot{i}} \oint h\left(z^{*}\right) z d z^{*} .
$$

Now Eq. (40) can be separated into two parts such that Eq. (42) may be applied to the first part and Eq. (43) to the second part. Thus

$W=\frac{J_{0}}{i} \oint \ln \left(\frac{\lambda_{0} \lambda-1}{\lambda-\lambda_{0}}\right) z_{0}^{*} d z_{0}-\frac{J_{0}}{i} \oint \ln \left(\frac{\lambda_{0}^{*} \lambda-1}{\lambda-\lambda_{0}^{*}}\right) z_{0} d z_{0}^{*}$.

A special case of Beth's result gives

$$
A=\frac{1}{2 i} \oint z_{0}^{*} d z_{0}=-\frac{1}{2 i} \oint z_{0} d z_{0}^{*}
$$


Hence Eq. (44) becomes

$$
W=\frac{V_{1}}{2 \pi} \frac{\oint \ln \left(\frac{\lambda_{0} \lambda-1}{\lambda-\lambda_{0}}\right) z_{0}^{*} d z_{0}}{\oint z_{0}^{*} d z_{0}}+\frac{V_{1}}{2 \pi} \frac{\oint \ln \left(\frac{\lambda_{0}^{*} \lambda-1}{\lambda-\lambda_{0}^{*}}\right) z_{0} d z_{0}^{*}}{\oint z_{0} d z_{0}^{*}} .
$$

Note that $W$ approaches the expression in Eq. (4) for sufficiently large $\left|\lambda_{0}\right|$ For reference also note that

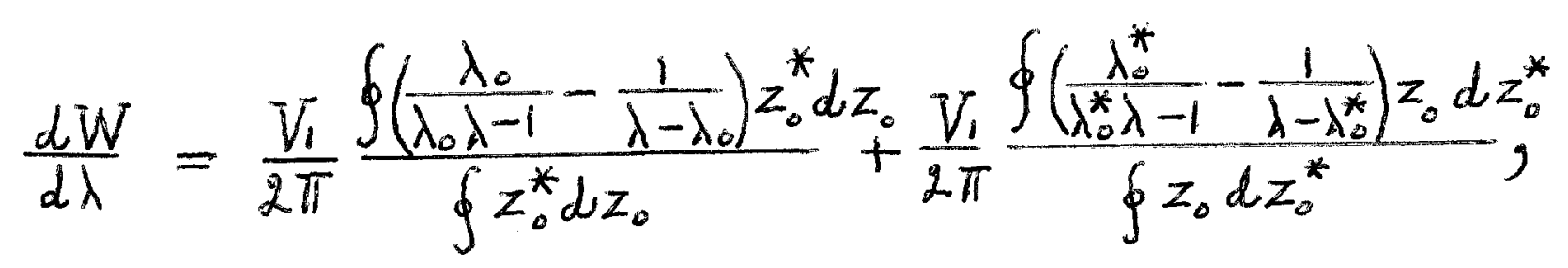

and

$$
\frac{d^{2} W}{d \lambda^{2}}=-\frac{V_{1}}{2 \pi} \frac{\oint\left[\left(\frac{\lambda_{0}}{\lambda_{0} \lambda-1}\right)^{2}-\left(\frac{1}{\lambda-\lambda_{0}}\right)^{2}\right] z_{0}^{*} d z_{0}}{\oint z_{0}^{*} d z_{0}}-\frac{V_{1}}{2 \pi} \frac{\oint\left[\left(\frac{\lambda_{0}^{*}}{\lambda_{0}^{*} \lambda-1}\right)^{2}-\frac{1}{\lambda-\lambda_{0}^{*}}\right)^{2} z_{0} d z_{0}^{*}}{\oint z_{0} d z_{0}^{*}}
$$

Equation (40) replaces Eq. (4) but the method remains the same except for the additional problem of determining $\lambda_{0}$ from specified locations $z_{0}$ on the boundary of the current block.

To begin then, $z_{0}$ is given for equaliy spaced intervals for each segment of the boundary. The corresponding $r_{0}$ are found using Eq. (2). Initial guesses for $w_{0}$ corresponding to $\lambda_{0}$ are made and $r_{0}$ found by integrating Eq. (6) from the origin. These $r_{0}$ are compared with the desired $r$. This change in $r_{p}$ is translated into a change in $w_{0}$ using the differential form of Eq. (6). In this manner improved values for $w_{0}$ are made and the process repeated until a convergence criterion is met.

Since Eq. (40) has replaced Eq. (4) the fields now become 
$-13-$

$$
H^{*}=i \frac{V_{0} C_{1}}{\pi q_{1}} G(s)\left(1+k z+\frac{1}{2} k^{\prime} z^{2}\right) C(\lambda),
$$

where

$$
C(\lambda)=\frac{\lambda}{2} \frac{\oint\left(\frac{\lambda_{0}}{\lambda_{0} \lambda-1}-\frac{1}{\lambda-\lambda_{0}}\right) z_{0}^{*} d z_{0}}{\oint z_{0}^{*} d z_{0}}+\frac{\lambda}{2} \frac{\oint\left(\frac{\lambda_{0}^{*}}{\lambda_{0}^{*} \lambda-1}-\frac{1}{\lambda-\lambda_{0}^{*}}\right) z_{0} d z_{0}^{*}}{\oint z_{0} d z_{0}^{*}} .
$$

The complex gradient becomes

$$
\begin{aligned}
\frac{d H^{*}}{d z}=i \frac{V_{0} C_{1}}{\pi q_{1}} G(s) & {\left[\left(k+k^{\prime} z\right) C(\lambda)+\left(1+k z+\frac{1}{2} k^{\prime} z^{2}\right)^{2} \frac{C_{1}}{g_{1}} G(s) \cdot\right.} \\
& \left.\left\{\sqrt{s^{2}-r^{2}} \frac{d \ln G G}{d s} C(\lambda)+\frac{\pi}{\phi_{1}}[C(\lambda)-D(\lambda)]\right\}\right],
\end{aligned}
$$

where

$$
D(\lambda)=\frac{\lambda^{2} \oint\left[\left(\frac{\lambda_{0}}{2}\right)^{2}-\left(\frac{1}{\lambda-\lambda_{0}}\right)^{2}\right]^{2} z_{0}^{*} d z_{0}}{\oint z_{0}^{*} d z_{0}}+\frac{\lambda^{2}}{2} \frac{\oint\left[\left(\frac{\lambda_{0}^{*}}{\left(\lambda_{0}^{*} \lambda-1\right.}\right)^{2}-\left(\frac{1}{\lambda-\lambda_{0}^{*}}\right)^{2}\right] z_{0} d z_{0}^{*}}{\oint z_{0} d z_{0}^{*}} .
$$

On the median plane Eqs. (49) and (51) give

$$
\begin{aligned}
& \frac{H_{y}^{\prime}(x, 0)}{H_{y}(0,0)}=\frac{G(\xi)}{G(r)} \frac{1}{1+C(1)}\left[\left(k+k^{\prime} x\right) C(\mu)\right. \\
& +\left(1+k x+\frac{1}{2} k^{2} x^{2}\right)^{2} \frac{C}{g_{1}} G(\xi)\left\{\left\{\sqrt{\xi^{2}-T^{2}} \frac{d}{d \xi} \ln G C(\mu)+\frac{\pi}{\phi_{1}}[C(\mu)-D(\mu)]\right\}\right] \text {. }
\end{aligned}
$$

(53) 
In general the parameter adjustment for $\rho_{0} \rho_{e} \alpha$ and $\tau$ proceeds as before except that Eq. (49) replaces Eq. (7) and Eq. (53) replaces Eq. (11). It has been noticed, however, that instabilities occur in taking numerical derivatives of Eq. (53). These instabilities are removed by turning on the change brought about by $C(\mu)$ and $D(\mu)$ very slowly. Thus for the $k^{\text {th }}$ iterate and using $C(\mu)$ as an example

$$
C_{\text {used }}^{(k)}=C_{\text {used }}^{(k-1)}+\left[C_{\text {calc }}^{(k)}-C_{\text {used }}^{(k-1)}\right] \cdot F A C(k),
$$

where

$$
F A C(k)=\tanh \left[\frac{.001}{A N(k)}\right]
$$

and $A N(k)$ is the root mean square fractional difference in the variables from the $(k-1)^{\text {st }}$ iterate. Since the iteration proceeds until AN is less than .0001 , the final $C_{\text {used }}$ will be very close to $C_{\text {calc. }}$

\section{REFERENCES}

1. M.H. Foss, Mathematical Techniques for Designing Field Shapes, Carnegie Inst. Tech., 1951 (NY0-911); see also: W. Hardt, DESY-Bericht A1.5 (1959), R. Perin, CERN Publ. AR/Int SG/64, Geneva, 1964, S. Jaidane, CEA-R-3238 (Rev.), Saclay, 1968.

2. The Preliminary Design of Beijing Proton Synchrotron, Beijing, China, 1979

3. W.R. Smythe, "Static and Dynamic Electricity", 3rd ed., McGrawHi11 Book Co., New York, 1968, p. 82. 
4. S.C. Snowdon, IEEE Trans. Nucl. Science, NS-18, 848 (1971).

5. W. Hardt, DESY-Bericht A1.5, Hamburg, 1959, p. 9.

6. R.A. Beth, Journ. App1. Phys. 38, 4689 (1967); also

R.A. Beth, Journ. App1. Phys. 40, 2445, 4782 (1969)

K. Halbach, Nucl. Instr. and Meth., 78, 185 (1970) 


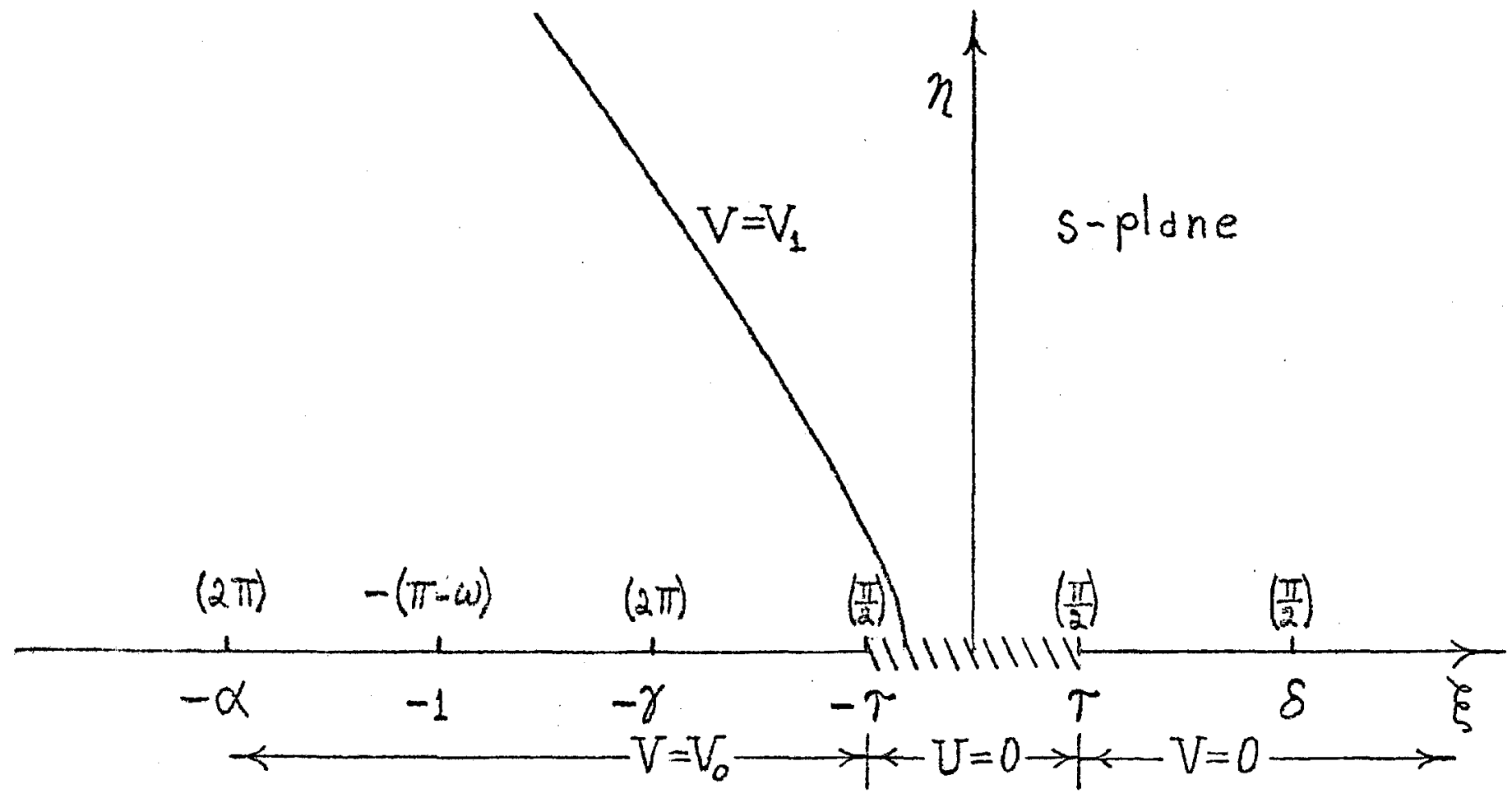

Figure 1. Initial Plane (generators on real axis)

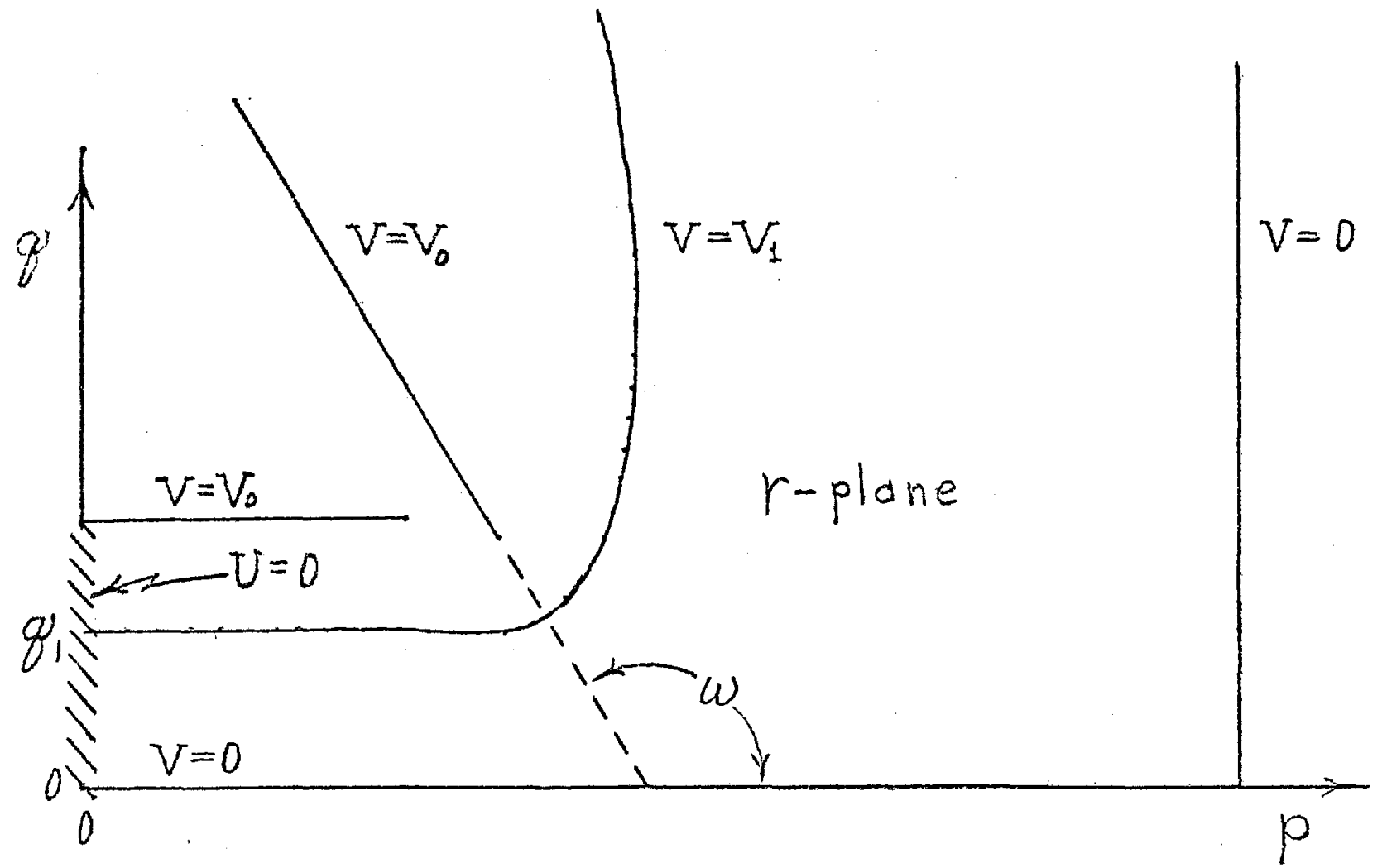

Figure 2. Transformed Plane (straight line generators) 


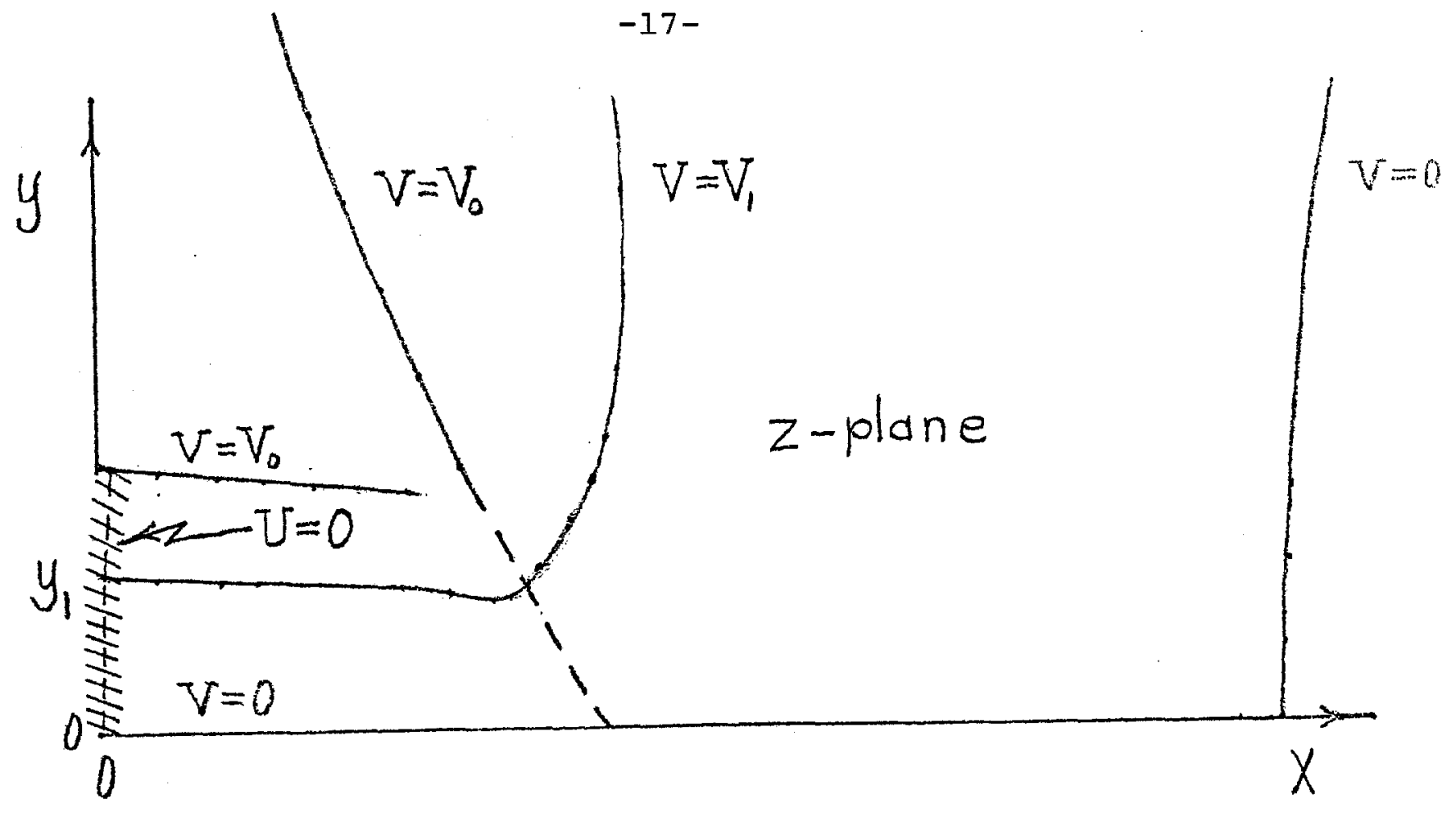

Figure 3. Conforma1 Mapping (generators distorted)

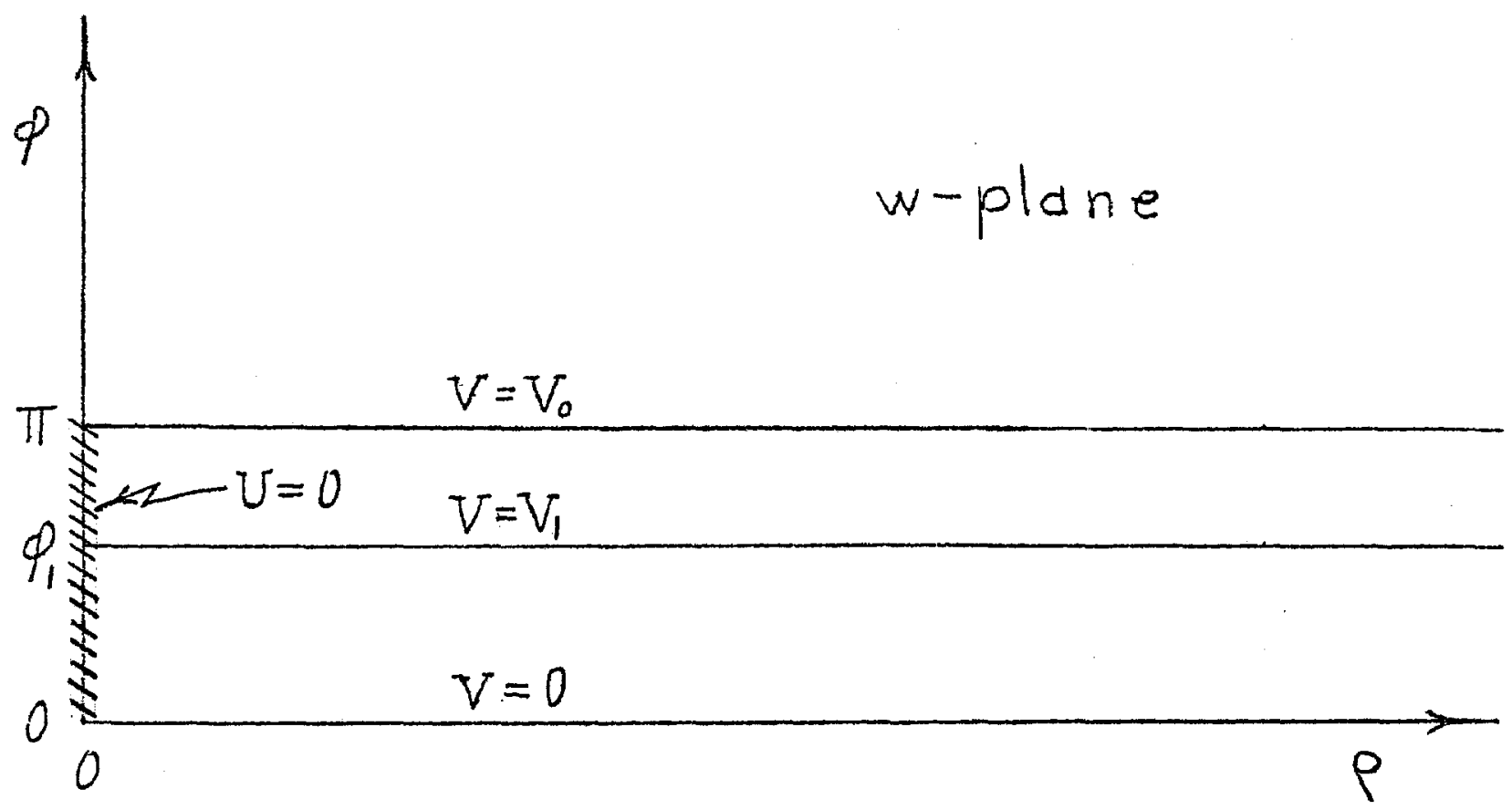

Figure 4. Intermediate Plane (real. axis is convenient stepping variable) 


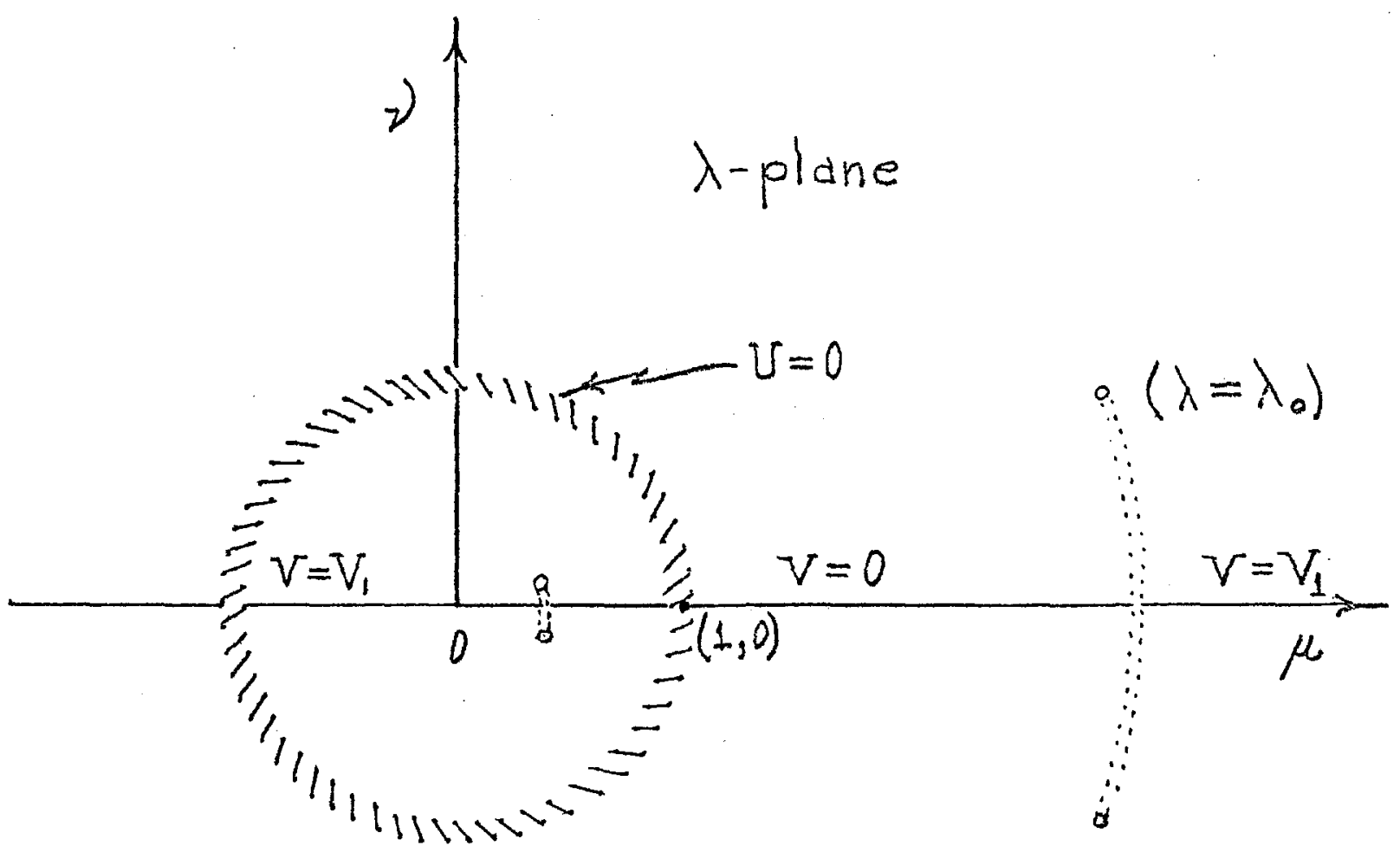

Figure 5. Intermediate Plane (used to specify magnetostatics) 